\title{
IMPACT OF CONCENTRATED COLLOIDAL SUSPENSION DROPS ON SOLID SURFACES
}

\author{
Volfango Bertola ${ }^{1 *}$, Mark Haw ${ }^{2}$ \\ 1 School of Engineering, University of Liverpool, Brownlow Hill, Liverpool, L69 3GH, UK \\ 2 Department of Chemical and Process Engineering, University of Strathclyde, 75 Montrose Street, \\ Glasgow, G1 1XJ, UK \\ *Corresponding Author. Phone: +44-151-7944804. Email: Volfango.Bertola@liverpool.ac.uk
}

\begin{abstract}
When droplets of high concentration wet powder impact on a solid surface, the large stresses that build up upon impact may convert them to a stable system of dry granules. Dilation/jamming has been proposed to explain such powder granulation processes. Stress causes dilation of particles into the droplet surface, against capillary pressure, roughening the surface on the scale of the constituent particles. Under the right conditions of stress magnitude and particle concentration, the droplet jams internally in response to capillary pressure, forming a mechanically stable granule. This remains a tentative model of granulation, which despite its importance in process industries ranging from minerals to foods to detergents, is still imperfectly understood.

This work presents the preliminary results of drop impact experiments of a suspension of near hard-core colloidal particles, with the purpose to investigate the impact morphology in the presence of shear thickening or jamming, which may be induced by the large velocity gradients arising upon drop impact. In particular, drops of a suspension of nearly hard-core particles in octadecene (volume fraction: $\sim 60 \%$ ) impacting on substrates of different wettability are studied experimentally by high-speed imaging, for impact Weber numbers ranging between 26 and 262. Upon impact, these drops do not exhibit inertial spreading, which is observed for other Newtonian and nonNewtonian fluid drops. On wettable surfaces (glass), impact is followed by capillary-driven spreading at the same rate observed in Newtonian fluids (Tanner's law), while on less wettable surfaces (PTFE) the colloidal suspension drops relax to achieve the shape of a spherical cap, but do not spread. This peculiar impact morphology, and in particular the absence of inertial spreading, is interpreted as a consequence of dilatancy and jamming occurring upon impact.
\end{abstract}

\section{KEYWORDS}

Colloidal suspensions, Hard-sphere interactions, Drop impact, Dilatancy, Jamming. 


\section{INTRODUCTION}

The impact of single and clouds of drops on a solid surface is of great scientific interest and practical importance. An improved understanding of drop impact is needed in applications as diverse as spray coating, spray painting, delivery of agricultural chemicals, spray cooling, ink-jet printing, soil erosion due to raindrop impact, turbine wear, soldering, powder granulation and even forensics (blood spatter). The phenomenology of macroscopic drop impact behaviour (shape deformation, spreading, rebound) can be described in terms of characteristic dimensionless groups such as the Reynolds number $\left(\operatorname{Re}=\rho u D_{0} / \mu\right)$, where $\rho$ is the fluid density, $u$ the impact velocity, $D_{0}$ the equilibrium drop diameter, and $\mu$ the dynamic viscosity), describing the relative roles of viscosity and inertia, and the Weber number $\left(\mathrm{We}=\rho \mathrm{u}^{2} \mathrm{D}_{0} / \sigma\right.$, where $\sigma$ is the surface tension of the fluid), which represents the competition between kinetic energy and surface energy. Since the pioneering observations of Worthington [1] more than 100 years ago many studies have been carried out on a range of fluids and surfaces, in a range of conditions [2],[3]. However, there remains limited fundamental understanding of the roles of fluid parameters and surface factors, especially in the practically most relevant case of fluids with complex non-Newtonian rheology. Non-Newtonian or so-called complex fluids [4] such as polymer solutions and melts, surfactant phases, suspensions, pastes and slurries, show stress-dependent response, e.g. shear- and extensional thinning and thickening, frequency-dependent viscoelasticity, significant normal stress differences, and history-dependent and geometry-dependent response. These rheological features are a consequence of mesoscopic or macromolecular-scale structure (polymers, micelles, colloids) with relaxation times comparable to the typical flow timescale: hence flow can generate significant mesoscopic structural deformation (e.g. aligned polymers, ordered colloid phases) taking systems far from mechanical equilibrium. In droplet impact, stress and strain rates also vary temporally and spatially throughout the event, therefore the structural/rheological response of the droplet can become exceptionally complex [5]-[8].

Particulate systems are an important class of non-Newtonian fluids. Particulates include colloidal suspensions, pastes and slurries (wet granulars), and emulsions (deformable particle systems), and are thus relevant across many deposition processes, such as spray-painting, surface treatment, soldering, and granulation. While dilute concentrations of colloids/particles $(<$ few $\%$ by volume) may have little rheological or dynamic effect beyond increase in effective viscosity and/or decrease in diffusion rate, highly concentrated particulates such as colloidal glasses and granular slurries show complicated rheology including yielding (the system behaviour is solid-like below a critical stress, or yield stress, and fluid-like above), jamming (the system behaviour is fluid-like below a critical stress, and solid-like above), and complex glassy dynamics [9],[10]. None of these 
phenomena are fully understood, but substantial research efforts over the past decade and significant advances in techniques such as direct observation by confocal microscopy, computational methods, and theoretical methods such as mode-coupling, soft glassy models, and shear-transformation zones [9]-[13], have seen a rapid improvement in basic understanding.

Jamming is often associated with dilation: as shown for granular media by Reynolds [14], and more recently investigated in colloids and pastes [15][16], above a critical concentration a system of particulates cannot deform macroscopically without local microscopic dilation, i.e. local decrease in particle volume fraction. When an incompressible solvent is involved, local dilation of the particle system requires either increase in local volume fraction elsewhere (and hence fluid flow) or, in the case of a droplet with a free surface, expansion of the total droplet volume may be possible by the particles deforming the surface. The dilation/jamming mechanism has been proposed to explain powder granulation processes where droplets of high concentration wet powder are converted by applied stress to a stable system of dry granules [17]. Stress causes dilation of particles into the droplet surface, against capillary pressure, roughening the surface on the scale of the constituent particles. Under the right conditions (stress, concentration) the droplet jams internally in response to capillary pressure, forming a mechanically stable granule. This remains a tentative model of granulation, which despite its importance in process industries ranging from minerals to foods to detergents, is still poorly understood.

Given this growing baseline of fundamental dynamics and rheological theory, there is now real potential to link droplet impact behaviour in particulates to fundamental system properties, via systematic quantitative data from controlled model experiments. Comprehensive, well-controlled study of droplet impact will feed back to better understanding of the consequences of glassy, yielding and jamming rheology. Furthermore, due to the high stresses and velocity gradients arising during impact, well beyond the maximum values achievable in conventional rheometric instruments, impacting drops represent a unique system to explore the phenomenology of yielding and jamming under extreme conditions.

This work presents the preliminary results of drop impact experiments of a suspension of near hard-core colloidal particles, with the purpose to investigate the impact morphology in the presence of shear thickening or jamming, which may be induced by the large velocity gradients arising upon drop impact. 


\section{EXPERIMENTAL METHOD}

\subsection{Material preparation and characterisation}

A model colloidal suspension characterised by nearly hard-core (i.e., purely repulsive) interactions was prepared with poly-methyl-methacrylate (PMMA, density: $1180 \mathrm{~kg} / \mathrm{m}^{3}$ ) spheres (radius $\mathrm{r} \sim 604 \mathrm{~nm}, 5 \%$ polydispersity) sterically stabilised by poly-12-hydroxy stearic acid (PHSA) chemically grafted onto their surface [18], and suspended in octadecene (density: $789 \mathrm{~kg} / \mathrm{m}^{3}$; surface tension: $0.03 \mathrm{~N} / \mathrm{m}$ ). Suspensions were centrifuged at $2000 \mathrm{rpm}$ for 12 hours to create a sediment, which was assumed to have a concentration of $64 \%$ (random close packing or maximum random packing) [19]. The sediment was then diluted with octadecene to a concentration of $\sim 60 \%$, corresponding to an average density of $1024 \mathrm{~kg} / \mathrm{m}^{3}$.

A correct choice of the fluid medium is essential to ensure that particles interact as hard spheres. In particular, the fluid must be a poor solvent for PMMA, in order to limit particles swelling, but a good solvent for PHSA so that polymer chains stretch out. When two particles come into contact, the repulsive energy given by the superposition of the two polymer layers rapidly increases as the distance between particles reduces [20]. Among the various suitable solvents, octadecene was selected because of its very low volatility.

According to the equilibrium thermodynamics phase diagram of ideal hard-spheres [21], for volume fractions between $54.5 \%$ and $74 \%$ (maximum crystalline close packing fraction) the system is crystalline. However, experiments showed that model suspensions of sterically-stabilized colloidal hard-spheres failed to crystallize for volume fractions greater than $58 \%$, remaining in a non-equilibrium phase known as the colloidal glass [22].

Figure 1 shows the flow curve for a dense hard-sphere suspensions at a volume fraction of $\sim 0.6$, obtained using a Thermo Mars II rotational rheometer equipped with stainless steel cone-plate geometry, in controlled-stress mode. At low shear rates, the fluid behaviour is essentially Newtonian (although there is a small yield stress, with magnitude of the order of $\sim 1 \mathrm{~Pa}$ ), with an apparent shear viscosity given by the ratio between the shear stress and the shear rate. However, a strong shear thickening can be observed at shear rates of the order of $\sim 3 \mathrm{~s}^{-1}$. Such shear-thickening could be an indication of jamming inside the fluid sample. Indeed, dilatancy and jamming can be directly related to shear-thickening during extensional rheology experiments [16]. The shearthickening part of the flow curve can be approximated by a power law, where coefficients are obtained by least-square best fit of experimental data:

$$
\tau_{x y}=0.038 \gamma^{5.225}
$$


For the sake of comparison, a Newtonian fluid with a viscosity of $\sim 1 \mathrm{~Pa} \cdot \mathrm{s}$ was obtained by preparing a $98 \%$ solution of glycerol in water (density: $1250 \mathrm{~kg} / \mathrm{m}^{3}$ ).

\subsection{Experimental apparatus and procedure}

Drops were generated using a syringe with blunt hypodermic needle (gauge 21, $0.495 \mathrm{~mm}$ i.d.) driven by a micrometric screw, and detached under their own weight. The needle was suspended above two substrates of different surface energy (glass and PTFE). To change the impact velocity, the drop release height was adjusted between 2 and $18 \mathrm{~cm}$ using a Vernier height gauge, which corresponds to theoretical free-fall velocities between 0.6 and $1.9 \mathrm{~m} / \mathrm{s}$. The competition between inertia and surface forces was characterised through the Weber number, We $=\rho v^{2} \mathrm{D}_{0} / \sigma$, where $\rho$ is the suspension density, $\sigma$ is the solvent surface tension, $\mathrm{v}$ is the drop velocity, and $\mathrm{D}_{0}$ the characteristic diameter before impact. The characteristic drop diameter before impact, measured by comparison with a known reference length, was $2.208 \pm 0.075 \mathrm{~mm}$, resulting in Weber numbers ranging from 26 to 262 .

A high-frame rate CMOS camera (Phantom v9000) equipped with a zoom lens (Navitar 7000) and horizontally aligned with the surface recorded the impacts of single drops. Back-to-front illumination was provided by a LED backlight (Philips AccentLed) which ensured a uniform illumination intensity, and images with a resolution of $576 \times 576$ pixels were captured at 4000 frames per second. Magnification was kept constant throughout all experiments and lengths on the image could be calculated by comparison with a reference length (spatial resolution: 16.26 $\mu \mathrm{m} /$ pixel). To ensure a fine optical alignment, the camera, the heated surface and the backlight were fixed to an optical breadboard. Quantitative data were extracted from images using proprietary software developed in LabView environment, which after background subtraction and image optimization measured the base diameter of drops, as well as the left and right contact angles using the drop profile tangent method.

Although the syringe-needle dispensing system described above ensures a very good reproducibility of the drop diameter, the particle concentration may not remain constant as the twophase (solid-liquid) mixture is pushed through the needle. In fact, the higher resistance encountered in the sudden contraction and in the needle is likely to induce self-filtration, favouring the flow of the suspending liquid with respect to particles [15]. When the mixture approaches the contraction the velocity of particles becomes smaller than that of the liquid (i.e., the liquid/solid slip ratio become greater than one); some of the particles accumulate in the corners of the contraction. Thus, the particle concentration in the drop is likely to be smaller than in the syringe reservoir, and slowly varies as the syringe reservoir is emptied. Moreover, the detachment of the colloidal suspension drop from the needle by capillary breakup, together with the slow relaxation time of the fluid, often 
results into a rupture of the filament at a certain distance from the drop, and consequently drops may exhibit a tail which is re-absorbed only after impact.

\section{RESULTS}

\subsection{Drop impact morphology}

The impact morphology of colloidal suspension drops on surfaces with different surface energy (glass and PTFE) is shown in Figures 2 and 3, for low $(\mathrm{We} \approx 20)$ and high $(\mathrm{We} \approx 200)$ impact kinetic energies, respectively. To allow a qualitative comparison with the impact behaviour of Newtonian fluids, images of glycerol solution drops (shear viscosity: $\sim 1 \mathrm{~Pa} \cdot \mathrm{s}$ ) impacting on the same target surfaces are also displayed.

These images suggest that at the moment of impact colloidal suspension drops are not necessarily spherical, but may exhibit deformations created at the moment of drop generation, similar to what happens during the generation of viscoplastic drops from capillary nozzles [23]. This can be observed more frequently for low falling heights (e.g., Figure 2), because the time between drop detachment and impact is too short for the drop to retrieve a spherical shape. Such deformations eventually disappear as the drop is deposited on the target surface and takes the shape of a spherical cap under the action of capillary forces.

These images also show that the inertial spreading of drops, which usually occurs in the first 5 ms after impact, is not noticeable on either surface, although one can observe capillary-driven spreading on the glass substrate at longer times after the impact. Spreading of colloidal suspension drops is however much slower than the spreading of Newtonian drops, because the fluid deviates from the Newtonian behaviour already at low shear rates (Figure 1).

When drops impact on the low-energy PTFE surface, one can observe an inertial deformation, comparatively smaller than the inertial deformation of Newtonian drops. After this inertial stage, drops seem to remain "frozen" on the surface, and do not exhibit further deformations, apart from a very small elastic recovery immediately after maximum spreading at high Weber numbers, as shown in Figure 3. This frozen state can be observed for several days, without any noticeable changes.

Because of the low volatility of octadecene, this cannot be due to the rapid evaporation of the solvent (which in fact continues to drive capillary spreading when drops impact on the glass surface). Instead, one can argue that the rapid inertial deformation upon impact induces dilatancy hence jamming in the colloidal suspension. If the jammed drop is on a glass surface, the capillary flow of the solvent is sufficient to drag some of the colloidal particles located near the boundary of the contact area (contact line) out of their position in the jammed structure; this propagates to the 
rest of the drop causing a re-fluidisation of the jammed system. However, if the drop is on a lowenergy surface such as PTFE, the solvent does not spread hence the jammed state can persist indefinitely.

Releasing a second drop a few seconds after the first impact, without removing the first drop from the substrate, does not lead to either drop coalescence (as expected for liquid droplets) or bouncing off (as expected for drops of solid-like materials). Drops remain permanently, stacked one on top of the other, as shown in Figure 4; in particular, the picture in Figure 4a was taken several days after the experiment, and that in Figure $4 \mathrm{~b}$ a few seconds after the second impact. Figure $4 \mathrm{~b}$ also shows that during the second impact only the drop on the top experiences a small inertial deformation, while the drop in contact with the substrate does not change its shape. This can be interpreted again in terms of jamming. When the second drop is released, the drop deposited on the surface is already jammed, therefore inertial deformation due to the impact between the two droplets can be observed only in the second drop.

\subsection{Drop spreading}

The base diameter (non-dimensionalised with respect to the characteristic drop diameter, $\mathrm{D}_{0}$ ) of colloidal suspension drops impacting on a glass surface is plotted as a function of time in Figure 5, for different impact velocities hence different Weber numbers. Upon impact, the drop base diameter has a modest inertial deformation for less than $1 \mathrm{~ms}$, then remains almost constant before the beginning of capillary-driven spreading.

Surprisingly, increasing the Weber number, i.e. the impact kinetic energy, reduces the inertial deformation; moreover, in the range of Weber numbers considered, at the end of inertial expansion the base diameter remains smaller than the drop equilibrium diameter. These observations suggest that upon impact the colloidal suspension jams, and therefore large inertial deformations are not possible; increasing the impact kinetic energy jamming is quicker, which further reduces the inertial deformation.

For longer times (> $100 \mathrm{~ms}$ ), irrespective of the Weber number, spreading continues, driven by capillary forces rather than inertia. In the capillary spreading of Newtonian drops, the base diameter grows at the rate $\mathrm{D} \sim \mathrm{t}^{1 / 10}$, as predicted by Tanner's law for Newtonian drops [24]. In the case of shear-thinning or shear-thickening fluids described by a power-law constitutive equation, the base diameter spreading rate is predicted by a more recent theoretical model [25] as:

$$
D \sim t^{p}
$$

where 


$$
p=\frac{n}{7+3 n}
$$

and $\mathrm{n}$ is the power-law exponent. Using the power-law exponent in Eq. (1), $\mathrm{n}=5.225$, from Eq. (3) one obtains a spreading exponent $\mathrm{p}=0.23$. In the case of the present experiments, for large times (i.e., well after the inertial stage) the base diameter scales as $\mathrm{D} \sim \mathrm{t}^{0.3}$, so that the scaling exponent $\mathrm{p}=$ 0.3), which is qualitatively consistent with the theoretical value given by Eq. (3).

Figure 6 shows the base diameter of colloidal drops impacting on a PTFE surface. The inertial deformation upon impact is again of very small amplitude and short duration (about $1 \mathrm{~ms}$ ). Unlike in the case of the glass surface, no capillary-driven spreading can be observed, and the base diameter remains constant for an indefinite time. It is interesting to observe that drops with different impact Weber numbers do not exhibit the tendency to attain a same equilibrium value of the base diameter, i.e. the value determined by the Young-Laplace equation, but seem to remain frozen (jammed) in the state they reach upon impact.

Figure 7 compares the base diameters of two colloidal suspension drops and two Newtonian drops with a viscosity of $\sim 1 \mathrm{~Pa} \cdot \mathrm{s}$ (i.e., a viscosity similar to that of the colloidal suspension before shear-thickening, see Figure 1). Despite the similar viscosity, the inertial spreading of the Newtonian drops is larger and has a longer duration (3-5 ms) than that of colloidal suspension drops; as expected, the drop deformation increases as the Weber number grows. This comparison provides further evidence that the impact on a solid substrate, even from a modest falling height, is able to cause jamming in colloidal suspension drops.

In the case of low-energy surfaces (PTFE), displayed in Figure 8, the comparison with Newtonian drops shows that, in addition to significant inertial deformations, Newtonian drops are also able to partially retract, in order to attain the equilibrium base diameter value. Note that the equilibrium value of the glycerol solution drops base diameter is significantly different from any of the colloidal suspension drop base diameters because the fluids have different surface tensions. Moreover, in colloidal suspension drops the base diameter (hence the contact angle) depends strongly on the impact Weber number, meaning that the contact angle cannot be determined using the Young-Laplace equation, but rather depends on the initial small inertial deformation and on particles jamming.

\section{CONCLUSION}

The impact of concentrated colloidal suspension drops onto solid surfaces was studied by highspeed imaging, and compared with Newtonian drops of similar viscosity. Experiments show that concentrated colloidal suspension drops do not exhibit large inertial deformations, which suggests 
that the impact induces dilatancy and jamming of colloidal particles, which prevent the drop deformation.

On glass substrates, at longer time scales one can observe capillary-driven spreading, where the spreading rate is about the same as that of Newtonian drops. On PTFE substrates, capillary spreading cannot be observed, and the drop base diameter remains constant after impact. The base diameter value depends on the impact Weber number, which suggests that wetting plays a negligible role in comparison to the initial inertial deformation and the subsequent jamming of the colloidal suspension.

\section{LIST OF SYMBOLS}

D Drop base diameter [m]

$\mathrm{D}_{0} \quad$ Drop equilibrium diameter $\quad[\mathrm{m}]$

n Constitutive equation index $[-]$

p Spreading exponent $\quad[-]$

$\mathrm{t}$ Time [s]

V Impact velocity $[\mathrm{m} / \mathrm{s}]$

We Weber number $\quad[-]$

$\sigma \quad$ Surface tension $\quad[\mathrm{N} / \mathrm{m}]$

$\rho \quad$ Density $\quad\left[\mathrm{kg} / \mathrm{m}^{3}\right]$

\section{ACKNOWLEDGEMENTS}

The Authors thank Andrew Schofield for particle synthesis.

\section{VITAE}

Dr. Volfango Bertola is Senior Lecturer in the School of Engineering, University of Liverpool, since 2011. He was previously Lecturer at the University of Edinburgh (2004-2011) and Marie Curie Research Fellow at the Ecole Normale Supérieure (ENS) in Paris (2001-2004). In 2009-2010 he was Visiting Professor at Politecnico di Torino (Italy). His research interests cover multiphase flows, complex fluid systems (polymer melts and solutions, gels, particles and colloidal suspensions, foams and emulsions), thermodynamics and heat transfer. He was awarded the Prize of the Italian Union of Thermo-Fluid Dynamics (UIT) in 2001. 


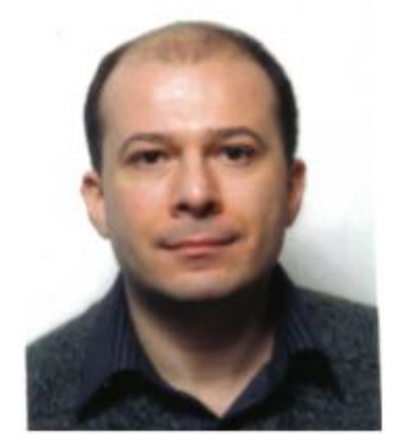

Dr Mark Haw is a Lecturer in the Department of Chemical and Process Engineering, University of Strathclyde. He was previously Lecturer at the University of Nottingham (2005-2008) having completed a PhD in Soft Matter Physics at the University of Edinburgh. His research covers colloidal and granular rheology, complex flows, phase behaviour and the role of interactions in aggregation, gelation and crystallisation. He is the author of a non-technical 'popular science' book on soft matter and Brownian motion, 'Middle World' (Macmillan, 2007).

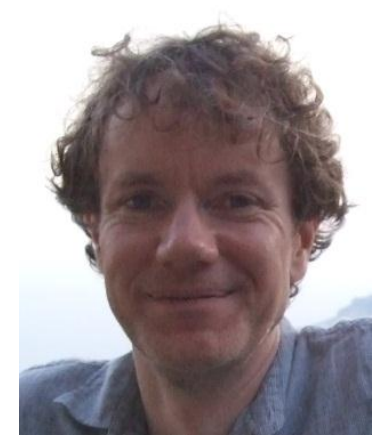

\section{REFERENCES}

[1] A.M. Worthington, On the form assumed by drops of liquids falling vertically on a horizontal plate, Proceedings of the Royal Society London, 25 (1876) 261-271.

[2] M. Rein, Phenomena of liquid drop impact on solid and liquid surfaces, Fluid Dynamics Research, 12 (1993) 61-93.

[3] A. L. Yarin, Drop impact dynamics: Splashing, spreading, receding, bouncing, Annu. Rev. Fluid Mech., 38 (2006), 159-192.

[4] R.G. Larson, The structure and rheology of complex fluids, OUP, New York, 1999;

[5] R. Crooks, D.V. Boger, $37 . \quad$ D. V. Boger, R. Crooks, Influence of fluid elasticity on drops impacting on dry surfaces, Journal of Rheology, 44 (2000): 973-996.

[6] V. Bertola, Drop impact on a hot surface: effect of a polymer additive, Experiments Fluids, 37 (2004) 653-664.

[7] J. Lampe, R. DiLalla, J. Grimaldi, J.P. Rothstein, Impact dynamics of drops on thin films of viscoelastic wormlike micelle solutions, Journal of Non-Newtonian Fluid Mechanics, 125 (2005) 11-23. 
[8] G. German, V. Bertola, Impact of shear-thinning and yield-stress drops on solid substrates, Journal of Physics: Condensed Matter, 21 (2009) 375111.

[9] G.L. Hunter, E. Weeks, The physics of the colloidal glass transition, Rep. Prog. Phys., 75 (2012) 066501.

[10]J. Olafsen (ed), Experimental and Computational Techniques in Soft Condensed Matter Physics, CUP, Cambridge, 2010.

[11]M.C. Jenkins, M.D. Haw, G.C. Barker, W.C.K. Poon, S.U. Egelhaaf, Does Gravity Cause Load-Bearing Bridges in Colloidal and Granular Systems?, Phys. Rev. Lett. 107 (2011) 038302.

[12] S.M. Fielding, M.E. Cates, P. Sollich, Shear banding, aging and noise dynamics in soft glassy materials Soft Matter 5 (2009) 2378-2382.

[13]M.L. Falk, J.S. Langer, Deformation and Failure of Amorphous, Solidlike Materials, Ann. Rev. Cond. Matt. Physics 2 (2011) 353-373.

[14] O. Reynolds, On the dilatancy of media composed of rigid particles in contact, Philos. Mag. Ser. 5, 50-20 (1885) 469.

[15]M.D. Haw, Jamming, two-fluid behaviour and self-filtration in concentrated colloidal suspensions, Physical Review Letters, 92 (2004) 185506.

[16] M.I. Smith, R. Besseling, M.E. Cates, Dilatancy in the flow and fracture of stretched colloidal suspensions, V. Bertola, Nature Comm. 1 (2010) 114.

[17] M.E. Cates, M.D. Haw, C.B. Holmes, Dilatancy, jamming and the physics of granulation, J. Phys. Condensed Matter, 17 (2005) S2517.

[18]L. Antl, J. W. Goodwin, R. D. Hill, R. H. Ottewill, S. M. Owens, S. Papworth, J. A. Waters, The preparation of poly(methyl-methacrylate) lattices in non-aqueous media, Colloids and Surfaces, 17, (1986) 67-78.

[19]J.D. Bernal, The bakerian lecture, 1962: The structure of liquids, Proceedings of the Royal Society A, 280 (1964) 290-321.

[20]B. A. de L. Costello, P. F. Luckham, Th. F. Tadros, Investigation of the interaction forces of polymer-coated surfaces using force balance, rheology, and osmotic pressure results, Langmuir, 8 (1992) 464-468.

[21]W.W. Wood, J. D. Jacobson, Preliminary results from a recalculation of the Monte Carlo equation of state of hard spheres, Journal of Chemical Physics, 27 (1957) 1207-1208.

[22]P.N. Pusey, W. van Megen, Phase behaviour of concentrated suspensions of nearly hard colloidal spheres, Nature, 320 (1986) 340-342. 
[23] G. German, V. Bertola, Formation of viscoplastic drops by capillary breakup, Physics of Fluids, 22 (2010) 033101.

[24]L.H. Tanner, The spreading of silicone oil drops on horizontal surfaces, J. Phys. D: Appl. Phys. 12 (1979) 1473-1484.

[25] V.M. Starov, A.N. Tyatyushkin, M.G. Velarde, S.A. Zhdanov, Spreading of non-Newtonian liquids over solid substrates, J. Colloid Interface Sci., 257 (2003) 284-290. 


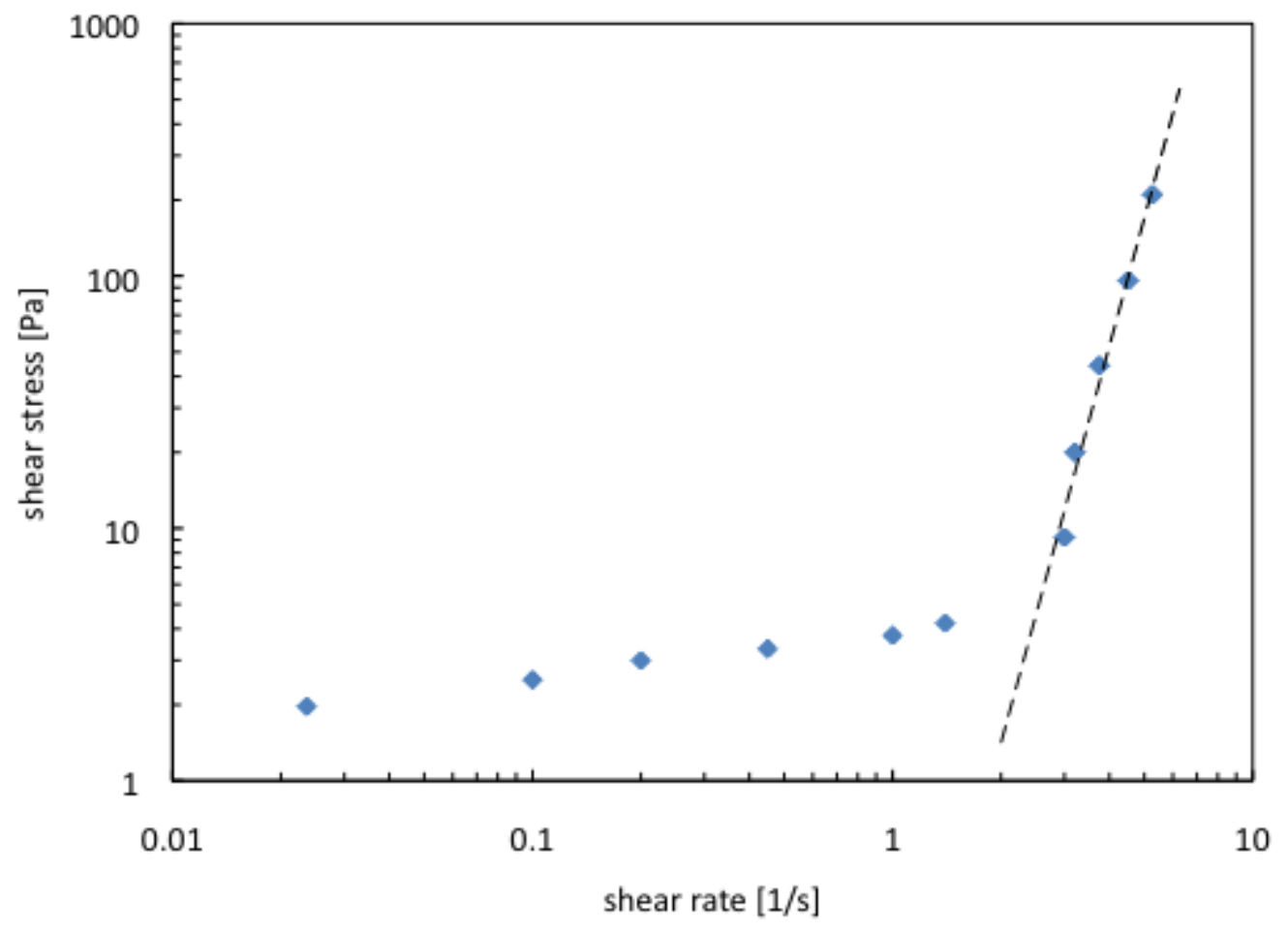

Figure 1. Flow curve of a colloidal suspension with volume fraction of 0.603 , measured in a rotational rheometer with cone-plate geometry at controlled stress. The dashed line represents a least-squares best fit to the last five points of the flow curve (Eq. 1). 


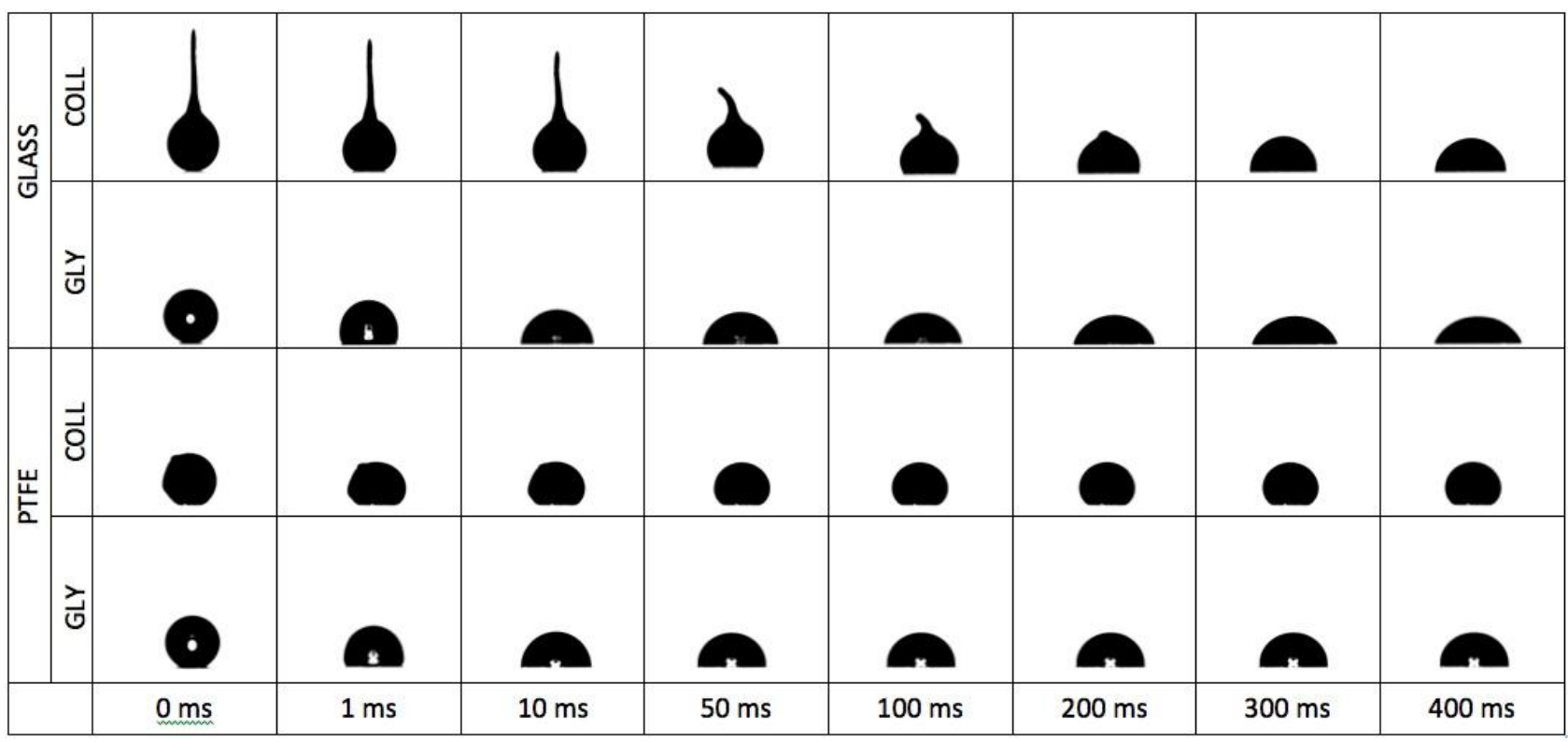

Figure 2. Drop impact morphology of colloidal suspension drops (COLL) impacting on glass and PTFE surfaces, and comparison with the impact morphology of high-viscosity ( 1Pa.s), Newtonian drop (GLY), for low impact kinetic energies (We 20). 


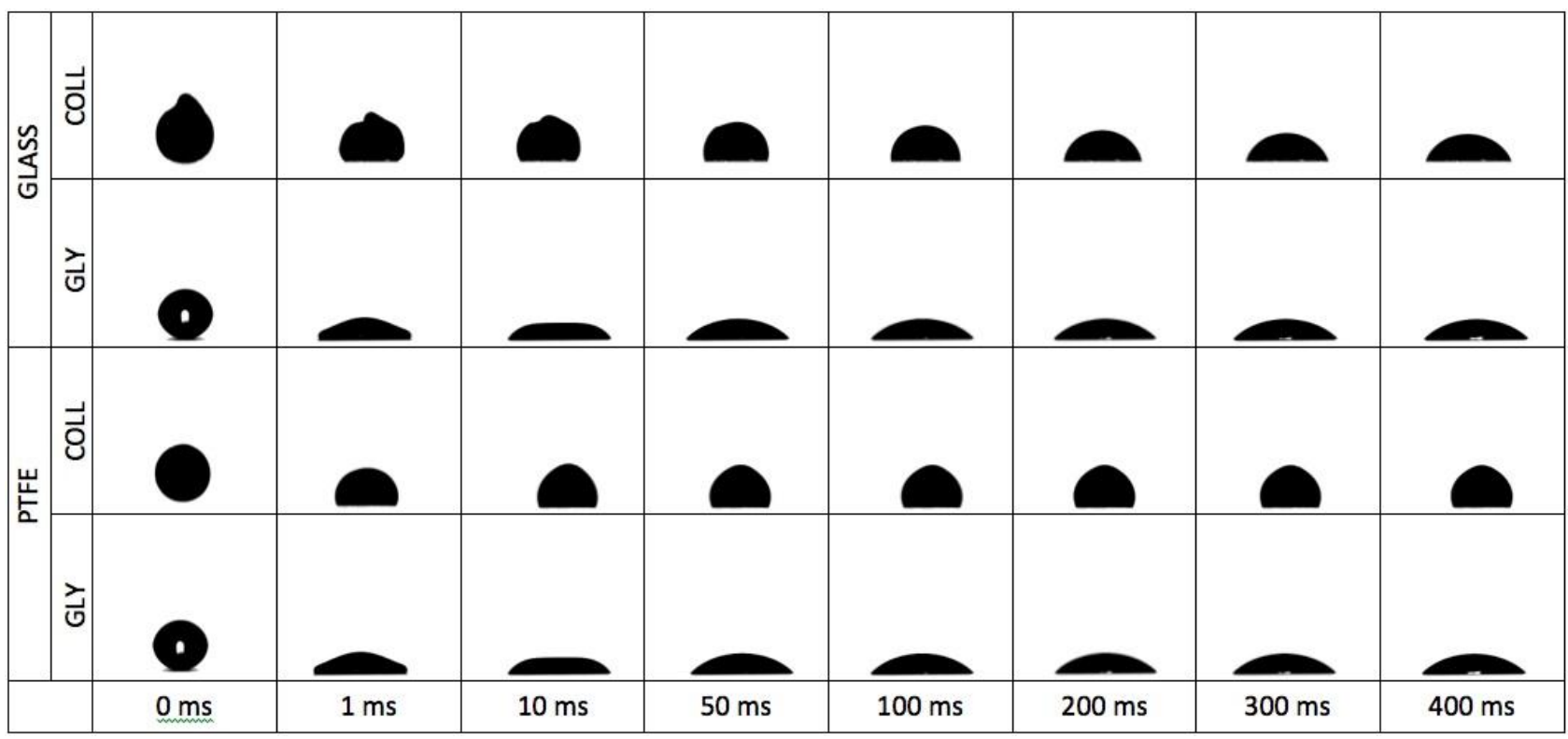

Figure 3. Drop impact morphology of colloidal suspension drops (COLL) impacting on glass and PTFE surfaces, and comparison with the impact morphology of high-viscosity ( 1Pa.s), Newtonian drop (GLY), for low impact kinetic energies (We 200). 

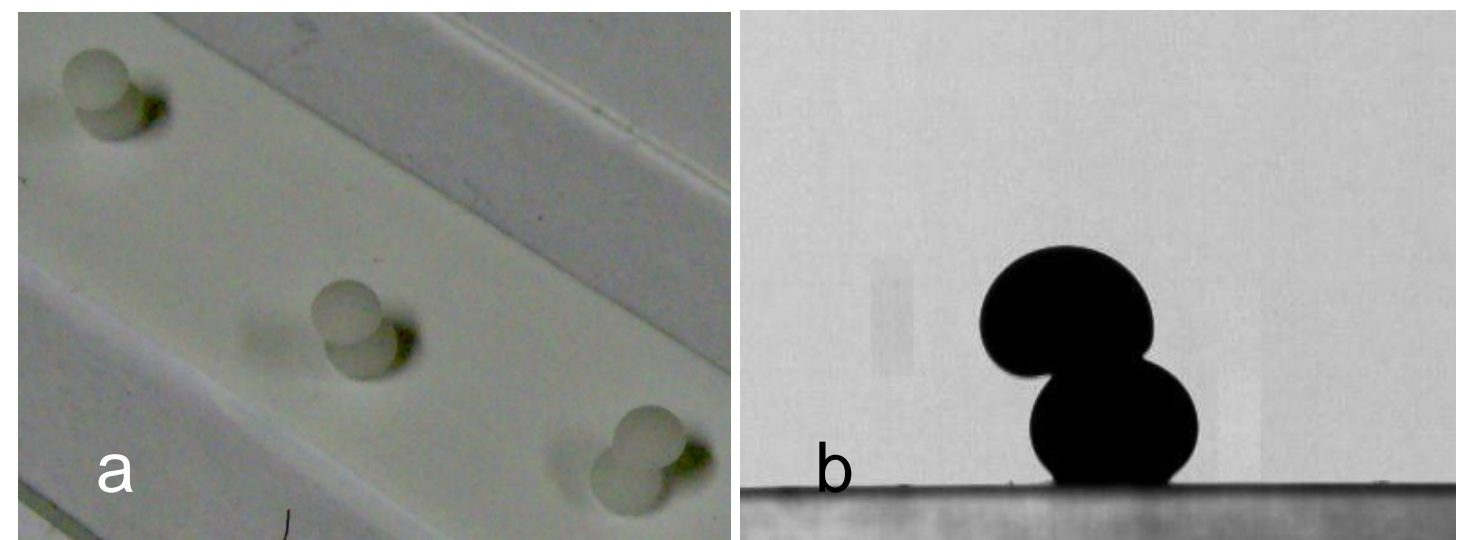

Figure 4. Jammed drop stacks on a PTFE surface, several days after the experiment (a) and a few second after the second impact (b). 


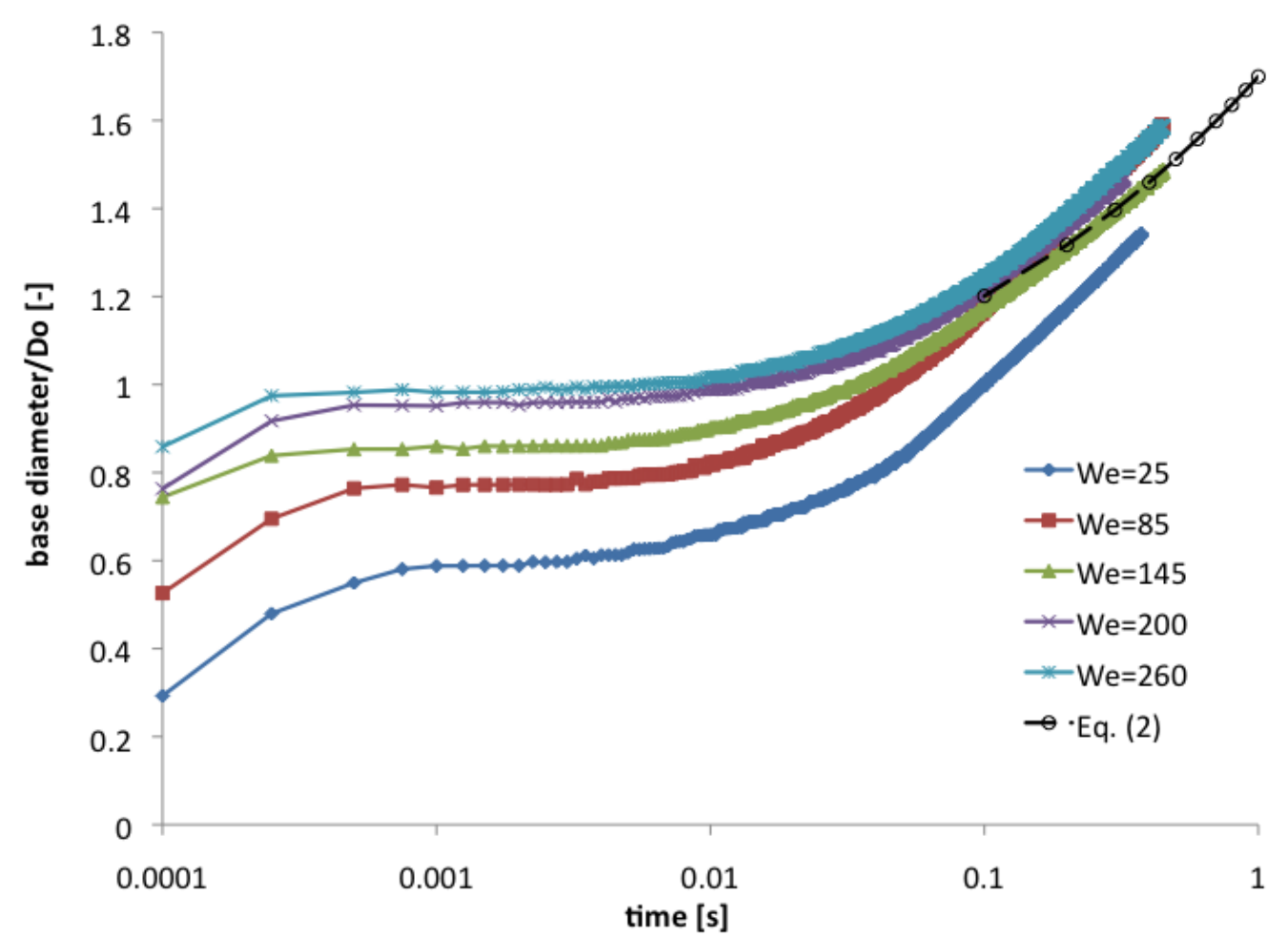

Figure 5. Dimensionless base diameter of colloidal suspension drops (volume fraction: $\sim 60 \%$ ) impacting on a glass surface, for different Weber numbers. The dashed line with open circles indicates the trend $\mathrm{D} / \mathrm{D}_{0} \sim \mathrm{t}^{0.3}$. 


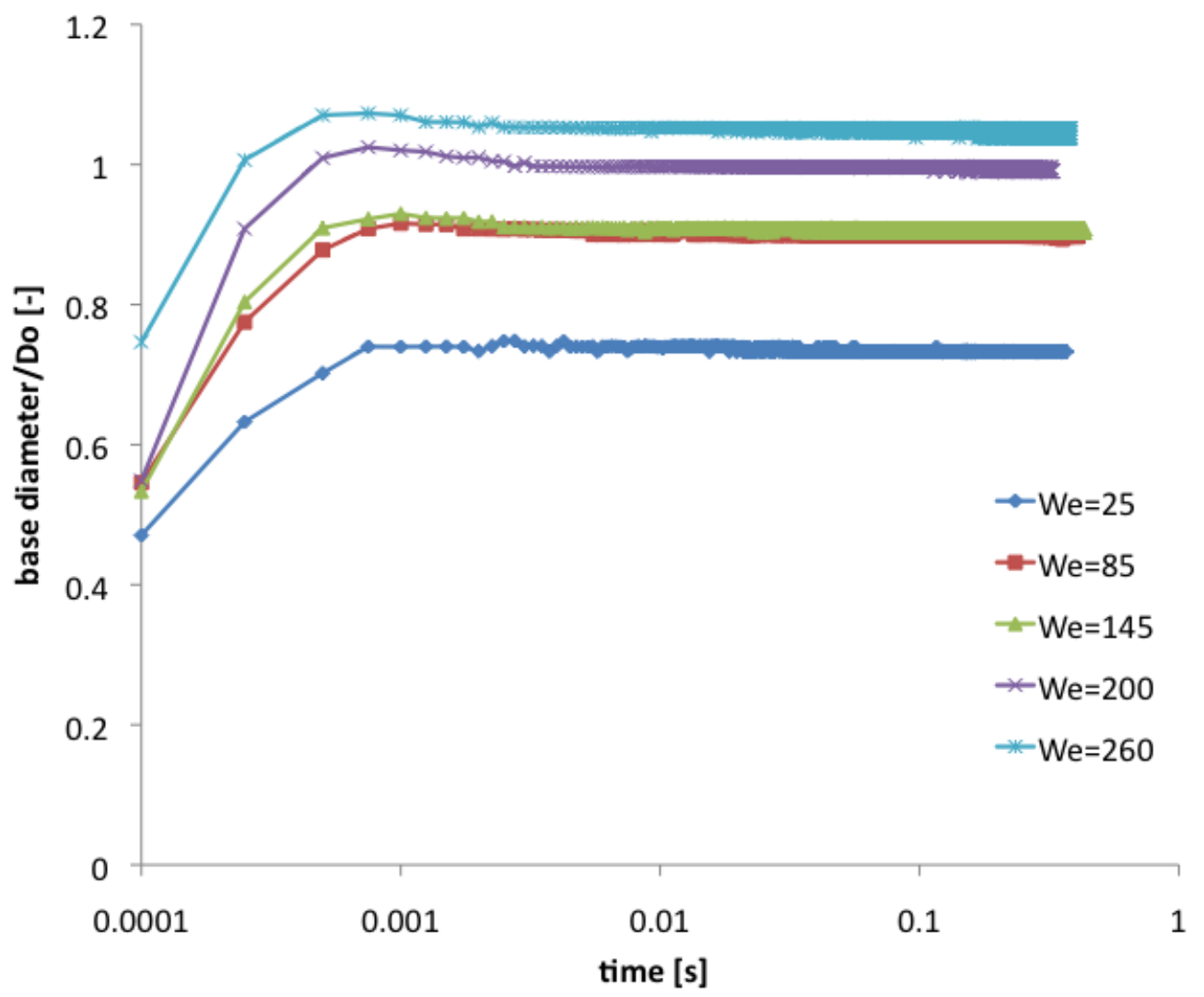

Figure 6. Dimensionless base diameter of colloidal suspension drops (volume fraction: $~ 60 \%$ ) impacting on a low-energy (PTFE) surface, for different Weber numbers. 


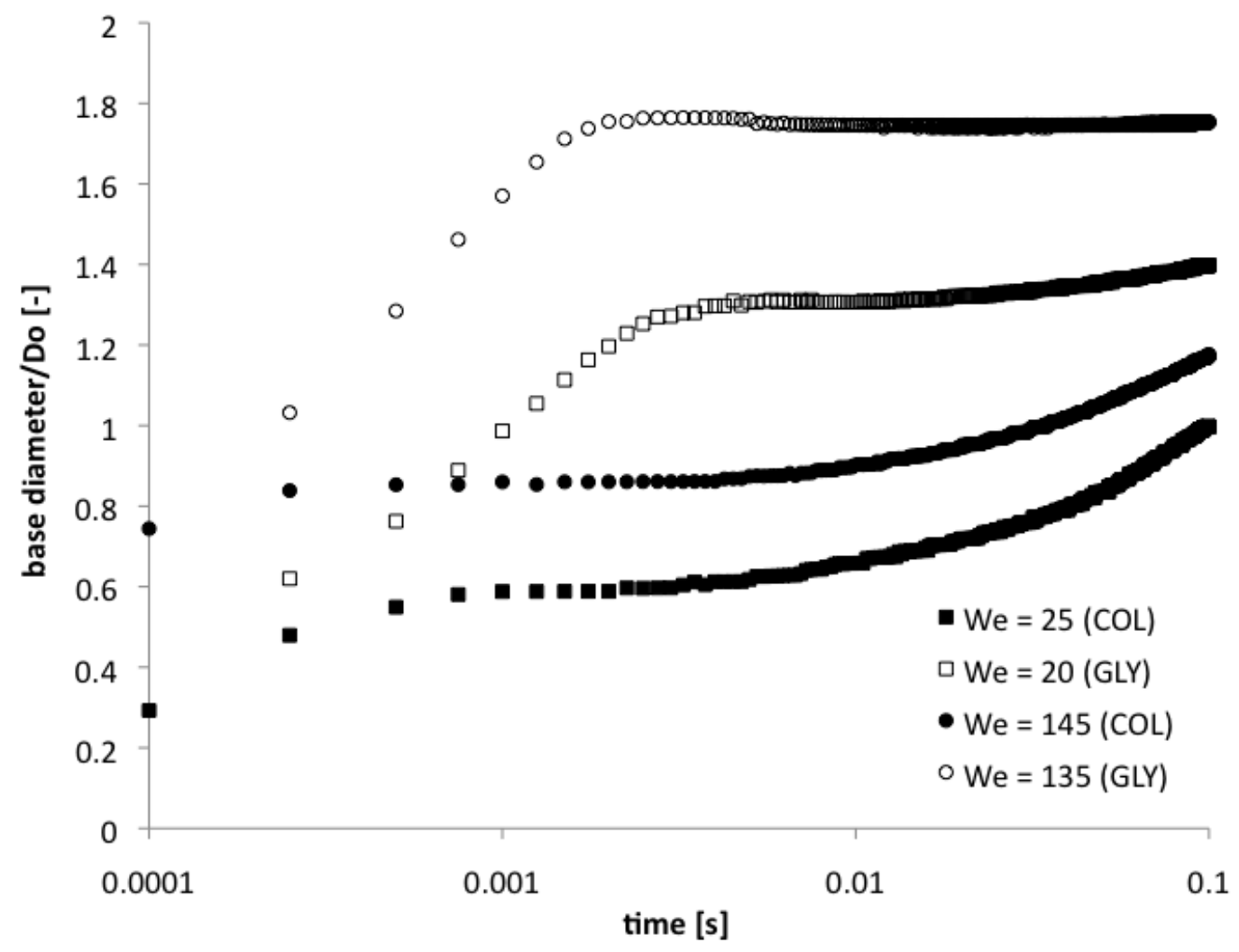

Figure 7. Comparison between the base diameters of colloidal suspension drops (filled symbols) and Newtonian drops (open symbols) impacting on glass. 


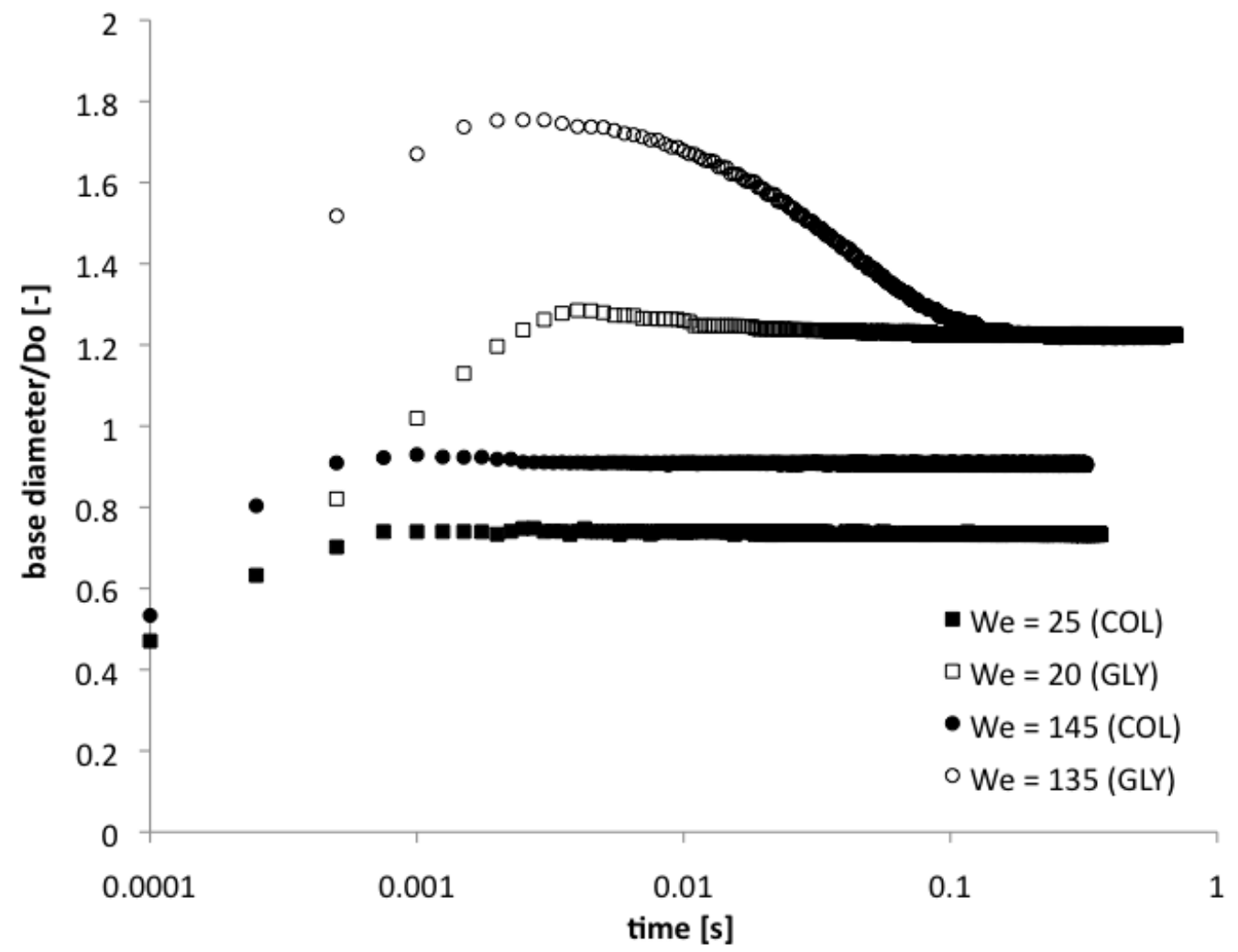

Figure 8. Comparison between the base diameters of colloidal suspension drops (filled symbols) and Newtonian drops (open symbols) impacting on PTFE. 